\title{
STUDY OF GROWTH KINETICS OF HIGH LIPID CONTENT ALGAE IN LOCAL ENVIRONMENT
}

\author{
Anika Ferdous ${ }^{1}$, Md. Mursalin Rahman Khandaker ${ }^{1,2}$, Farid Ahmad ${ }^{3}$, John Liton Munshi', \\ and Mohidus Samad Khan ${ }^{1,2 *}$ \\ ${ }^{1}$ Department of Chemical Engineering, Bangladesh University of Engineering and Technology, \\ Dhaka, Bangladesh \\ ${ }^{2}$ Environmental Sustainability in Textile Industries (ESTex), Department of Chemical Engineering, \\ Bangladesh University of Engineering and Technology, Dhaka, Bangladesh \\ ${ }^{3}$ Institute of Appropriate Technology (IAT), Bangladesh University of Engineering and Technology, \\ Dhaka, Bangladesh \\ ${ }^{4}$ Bangladesh Council of Scientific and Environmental Research (BCSIR), Dhaka, Bangladesh.
}

\begin{abstract}
In recent years algae has been receiving a lot of interest as an alternate source of biofuel. Fast growth and rich lipid content make algae a great candidate for producing eco-friendly and non-toxic biodiesel. The strain Chlorella vulgaris, has shown great potential as a source of oil. To optimize the mass culture of microalgae Chlorella vulgaris, it is necessary to develop growth kinetic model of Chlorella vulgaris. This work presents a comparative study on growth kinetics of Chlorella vulgaris in batch cultures in four different media, namely- $\mathrm{CH}$, modified $\mathrm{CH}$, Bold's Basal (BB), and modified Bold's Basal (BB). Spectroscopic analysis was supported by visual change of color in the algal biomass solution and the microscopic imaging, and was validated with dry cell mass measurement in this study. The data were analyzed to produce growth curves to find exponential phase for each medium. Exponential phases are the best period to harvest the culture. Hence the duration of exponential phase and the final biomass concentration at the end of exponential phase are two important parameters to determine the most suitable medium for mass culture. In this study the overall specific growth, doubling time and biomass productivity of Chlorella vulgaris for $\mathrm{CH}$, modified $\mathrm{CH}, \mathrm{BB}$ and modified $\mathrm{BB}$ media were measured. This study will help to find a suitable media to culture Chlorella vulgaris, a high lipid content algae in the local environment. The information can be used to design a system for mass culture of the strain for biodiesel production.
\end{abstract}

Keywords: Algae; biodiesel; growth kinetics; optical density; dry cell weight; exponential phase; batch culture

\section{Introduction}

The depletion of fossil fuel supplies and the contribution of these to the accumulation of $\mathrm{CO}_{2}$ in the atmosphere make it a priority to search for renewable and carbon neutral transport fuels for environmental and economic sustainability [1]. Microalgae have the capacity to fixate inorganic $\mathrm{CO}_{2}$ into organic matters. A desirable characteristic of many species of microalgae is the capability of producing and accumulating lipids suitable for biodiesel. Some algal species can accumulate polysaccharides as starch for bioethanol purpose [2]. Microalgae have a shorter average harvesting cycle of around 10 days, while traditional oil crops are only harvested once or twice a year.

The cost of biodiesel could be reduced if one considers non-edible source such as microalgae oil [3, 4]. Different studies have shown Chlorella to have potential as a source for oil in the production of bio- diesel $[5,6]$. Finding algae strains to grow is not as difficult as cultivating a specific strain of algae for biodiesel production. A class of biomolecules synthesized by many species of microalgae is the neutral lipids, or triacylglycerol (TAGs) and their content in microalgae varies from organism to organism. Some microalgae can produce more than $60 \%$ of their dry cell weight in the form of lipids under certain conditions [7, 8]. Table 1 enlists different algal strains with their lipid contents [9].

Chlorella is a microorganism of interest in this work due to its growth rate and lipid content. Chlorella can be classified as a single cell and green algae. Chlorella cells make wide range of lipid during their growth cycle. It is reported to be in the range of $22.1-$ $56.6 \%$ of the dry biomass. Some properties, apart from containing high lipid that make Chlorella vulgaris appropriate for use in food industry, aquaculture, cosmetics, pharmaceuticals, waste water treatment and the production of biofuel, are- 
- Rapid growth

- $\quad$ Easy and flexible terms of culture

- Resistance against interfering factors

Table 1

Lipid content of some microalgae

\begin{tabular}{|c|c|}
\hline Species & Lipid (\% dry matter) \\
\hline Botryococcus braunii & $25-75$ \\
\hline Chlorella vulgaris & $22.1-56.6$ \\
\hline Chlorella sorokiniana & 19.3 \\
\hline Chlorella protothecoides & $23 / 55$ \\
\hline Chlorella minutissima & 57 \\
\hline Dunaliella bioculata & 8 \\
\hline Dunaliella salina & $14-20$ \\
\hline Neochloris oleoabundans & $35-65$ \\
\hline Spirulina maxima & $4-9$ \\
\hline
\end{tabular}

A culture growing in a closed system where nothing is added or taken away is called a batch culture. An inoculum of cells is added and the batch culture is kept in an environment, which favors growth. Under a typical homogenous batch regime (in a closed system), microalgae will pass through the following growth phases illustrated in Figure 1 [10].
A. Adaptation (lag phase)
B. Exponential growth phase (log phase)
C. Stationary phase
D. Logarithmic death phase

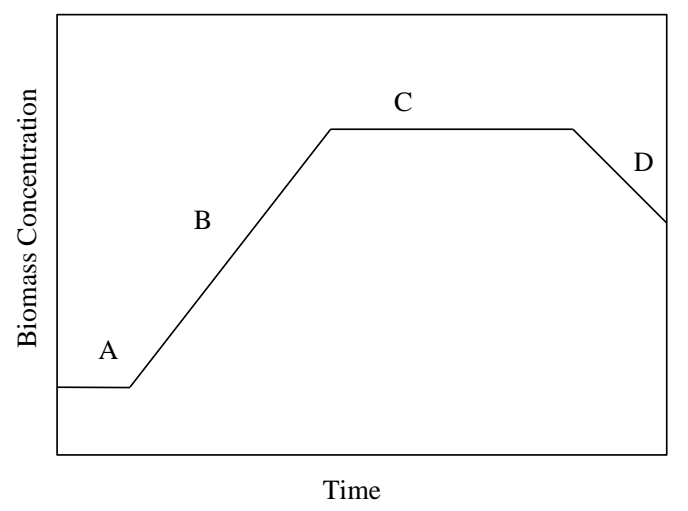

Fig. 1 Microalgae batch growth profile [10]

Cells growing by division in a log phase, synonymously exponential phase, are often in their healthiest state where one cell divides into two new cells and so on. If the number of cells is plotted in a semi logarithmic way a straight line will be seen. Exponential growth cannot grow indefinitely in a batch culture and only occurs in the log phase.

In view of microalgal system optimization it is essential to have insight in the kinetics related to the microalgal growth. There are different methods of estimating biomass and growth where optical density (OD), dry weight (DW) and cell count are commonly used methods. Dry weight and cell counting are time consuming methods whilst OD measurements are faster. For unicellular microorganisms optical density is proportional to cell count and dry weight, within certain limits [2]. Using various measurement techniques, this study was conducted to investigate how nutrient media composition can affect the growth in a specific, controlled environment.

This study investigates the growth of Chlorella vulgaris, a high lipid content algae, in local environment in batch cultures in four different media, namely- $\mathrm{CH}$, modified $\mathrm{CH}$, Bold's Basal (BB), and modified Bold's Basal (BB). Spectroscopic analysis was supported by visual change of color in the algal biomass solution and was validated with dry cell mass measurement in this study. The obtained data were later analyzed to determine four parameters- duration of exponential phase, overall specific growth rate, doubling time and volumetric biomass yield. Finally, these four parameters were compared to find the most suitable media to grow Chlorella vulgaris in the local environment.

\section{Materials and Methods}

\subsection{Microalgae culture}

In this study the microalgae strain that was studied is Chlorella vulgaris. It was collected from Commonwealth Scientific and Industrial Research Organization (CSIRO), Australia. Figure 2 shows a microscopic image of a matured Chlorella vulgaris cell.

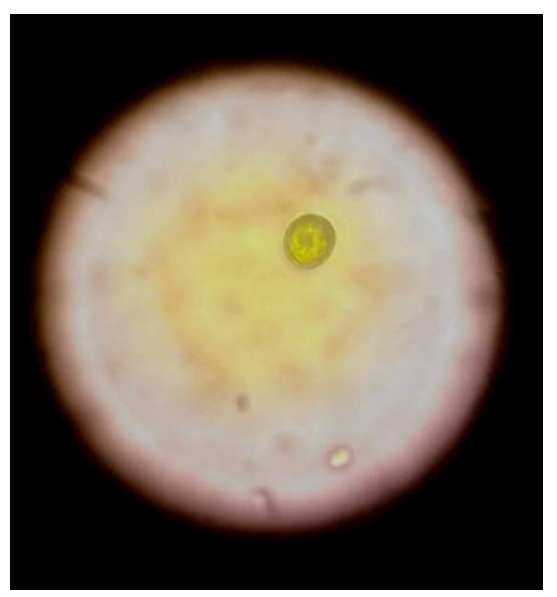

Fig. 2 Microscopic image of a matured Chlorella vulgaris cell

The growth kinetics of Chlorella vulgaris was studied in four different nutrient media namely- $\mathrm{CH}$ medium, Modified CH medium, Bold's Basal (BB) medium and Modified BB medium. Bold's Basal (BB) medium is a popular medium for growing microalgae whereas $\mathrm{CH}$ (short for Chlorella) is a locally developed medium. 
$\mathrm{CH}$ medium was prepared using following chemicals- $\mathrm{MgSO}_{4} .7 \mathrm{H}_{2} \mathrm{O}(10.4 \times 10 \mathrm{mg} / \mathrm{L}), \mathrm{K}_{2} \mathrm{HPO}_{4}$ $(40.4 \times 10 \mathrm{mg} / \mathrm{L}), \mathrm{KNO}_{3}(1.08 \mathrm{~g} / \mathrm{L}), \mathrm{FeSO}_{4} .7 \mathrm{H}_{2} \mathrm{O}$ $(5 \times 10 \mathrm{mg} / \mathrm{L})$ and $\mathrm{CaSO}_{4} \cdot 2 \mathrm{H}_{2} \mathrm{O}(2 \times 10 \mathrm{mg} / \mathrm{L})$ Modified $\mathrm{CH}$ medium was made following the same recipe, except it contained a mixture of three vitamins- Biotin, Vitamin $\mathrm{B}_{12}$ and Thiamin $\mathrm{HCl}$.

$\mathrm{BB}$ medium was prepared using $\mathrm{MgSO}_{4} .7 \mathrm{H}_{2} \mathrm{O}(7.5$ $\mathrm{g} / \mathrm{L}), \mathrm{NaNO}_{3}(25 \mathrm{~g} / \mathrm{L}), \mathrm{K}_{2} \mathrm{HPO}_{4}(7.5 \mathrm{~g} / \mathrm{L}), \mathrm{KH}_{2} \mathrm{PO}_{4}$ (17.5 g/L), $\mathrm{H}_{3} \mathrm{BO}_{3}(11.42 \mathrm{~g} / \mathrm{L}), \mathrm{CaCl}_{2} .2 \mathrm{H}_{2} \mathrm{O}(2.5 \mathrm{~g} / \mathrm{L})$, $\mathrm{FeSO}_{4} .7 \mathrm{H}_{2} \mathrm{O}(4.98 \mathrm{~g} / \mathrm{L})$, EDTA $(50 \mathrm{~g} / \mathrm{L}), \mathrm{KOH}(31$ $\mathrm{g} / \mathrm{L}), \quad \mathrm{H}_{2} \mathrm{SO}_{4} \quad(1 \mathrm{ml} / \mathrm{L}), \quad \mathrm{MnCl}_{2} .4 \mathrm{H}_{2} \mathrm{O} \quad(1.44 \mathrm{~g} / \mathrm{L})$, $\mathrm{CuSO}_{4} .5 \mathrm{H}_{2} \mathrm{O}(1.57 \mathrm{~g} / \mathrm{L}), \mathrm{ZnSO}_{4} .7 \mathrm{H}_{2} \mathrm{O}(8.82 \mathrm{~g} / \mathrm{L})$, $\mathrm{MoO}_{3}(0.71 \mathrm{~g} / \mathrm{L})$, and $\mathrm{Co}\left(\mathrm{NO}_{3}\right)_{2} .6 \mathrm{H}_{2} \mathrm{O}(0.49 \mathrm{~g} / \mathrm{L})$. Modified BB medium contained the same compounds as BB medium, except it contained an additional mixture of three vitamins- Biotin, Vitamin $\mathrm{B}_{12}$ and Thiamin $\mathrm{HCl}$.

The microalgae were cultured in $250 \mathrm{ml}$ Erlenmeyer flasks. Different batches of the experiments for each medium were conducted in series. The flasks were first filled with $200 \mathrm{ml}$ nutrient medium and then autoclaved for 20 minutes. After cooling down to the room temperature, each flask was inoculated with $5 \mathrm{ml}$ of algae stock solution. The cultures were mixed by means of sterile air in a laminar airflow chamber. The flasks were kept in a plant-growth room having a controlled temperature of $25 \pm 2^{\circ} \mathrm{C}$, and lit with $1200 \mathrm{lux} / \mathrm{m}^{2}$ fluorescent light with a photoperiod of 8 hours a day. Figure 3 shows the experimental setup of the study.

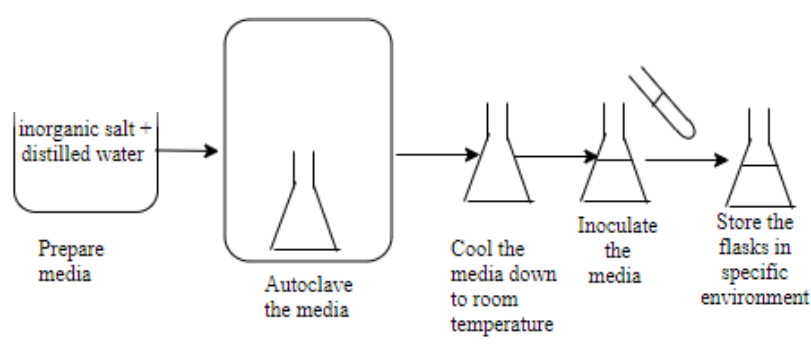

Fig. 3 Experimental setup for growth kinetic study

\subsection{Biomass quantification}

The parameters observed for quantifying the biomass are- absorbance / optical density and dry cell weight of the biomass. After substantial growth period a portion of the algae biomass was separated and analyzed for measurement of optical density The study was continued for 30 days and samples were collected on day $3,6,9,12,15,18,21,24,27$ and 30 . Then the solution of biomass was dried to find the mass of biomass present in the specific solution. Spectroscopic analysis was supported by visual change of color in the algal biomass solution and was validated with dry cell mass measurement in this study.
HACH Spectrophotometer, DR-6000 was used to measure the optical density of the microalgae cultures. For this study, the optimal wavelength used was 678 $\mathrm{nm}$, which was determined based on the UV spectrum [11]. Optical Density of the samples were measured at a regular time interval for 30 days.

For this step, $10 \mathrm{ml}$ of cultivated sample was collected from each media and initially the Total Dissolved Solid (TDS) value was measured for each sample. Then the samples were transferred to clean beakers, which were bone dried earlier. Before transferring the samples, the weight of the dry, empty beakers was recorded. The beakers were then put inside a desiccator to be heated around $80 \mathrm{oC}$ overnight. This removed any moisture content from the sample and later the weight of the dried sample along with the beaker was calculated. Assuming all the dissolved solids left with moisture, leaving behind only the suspended solids, which were dried Chlorella vulgaris, dry cell weight was calculated from the collected data, using the following equation -

$$
\begin{aligned}
\mathrm{DCW}= & (\text { weight of beaker }+ \text { solid })-\text { weight of dry } \\
& \text { beaker-TDS }
\end{aligned}
$$

\subsection{Determination of growth kinetics parameter}

In this study, four decisive parameters, obtained from the growth kinetics of microalgae, were measured, namely- duration of exponential phase, overall specific growth rate, doubling time and volumetric biomass yield or biomass productivity.

Duration of exponential phase was measured from the growth curves.

Since the curve of the exponential growth phase is constantly increasing it is difficult to find a "straight" line portion to measure, which consequently complicates finding a single growth rate for the growth period. One solution is to measure an overall specific growth rate, which is measured using the following formula [12]-

$$
\mu_{\text {overall }}=\frac{\log 10 N_{\text {end }}-\log 10 N_{o}}{t_{\text {end }}-t_{o}}
$$

where, $\mu_{\text {overall }}=$ Overall specific growth rate

$t_{\text {end }}-t_{o}=$ Duration of study period

$N_{\text {end }}=$ Biomass concentration at the end of study period

$N_{o}=$ Biomass concentration at the beginning of study period.

The doubling time is simply the time (in hours) required for the cells to divide. A large doubling time value means slow growth, while a small doubling time value means rapid growth. Doubling time $(\mathrm{G})$ can be calculated using the following formula [12]:

$$
\mathrm{G}=\frac{\log 10_{2}}{\mu_{\text {overall }}} \times 24
$$


Biomass productivity for each culture was measured using the following formula [12]-

$$
P_{\text {Biomass }}\left(g L^{-1} d^{-1}\right)=\left(X_{2}-X_{1}\right) \cdot\left(t_{2}-t_{1}\right)^{-1}
$$

Where, $X_{1}$ and $X_{2}$ are dry cell weight concentrations of Chlorella vulgaris in $\mathrm{gL}^{-1}$ on days $t_{l}$ (start point of cultivation) and $t_{2}$ (endpoint of cultivation) respectively.

\section{Results and Discussions}

\subsection{Growth curves}

Growth curves are a visual representation of the growth of microorganisms in the media. Microorganism growth curves follow Monod model [10]. In this study the growth curves are obtained by putting cell concentration against time. Growth curves show that after a lag phase, the microalgae cells grow exponentially to reach a phase of declining relative growth. Figure 4 shows growth curves obtained for Chlorella vulgaris in four media.
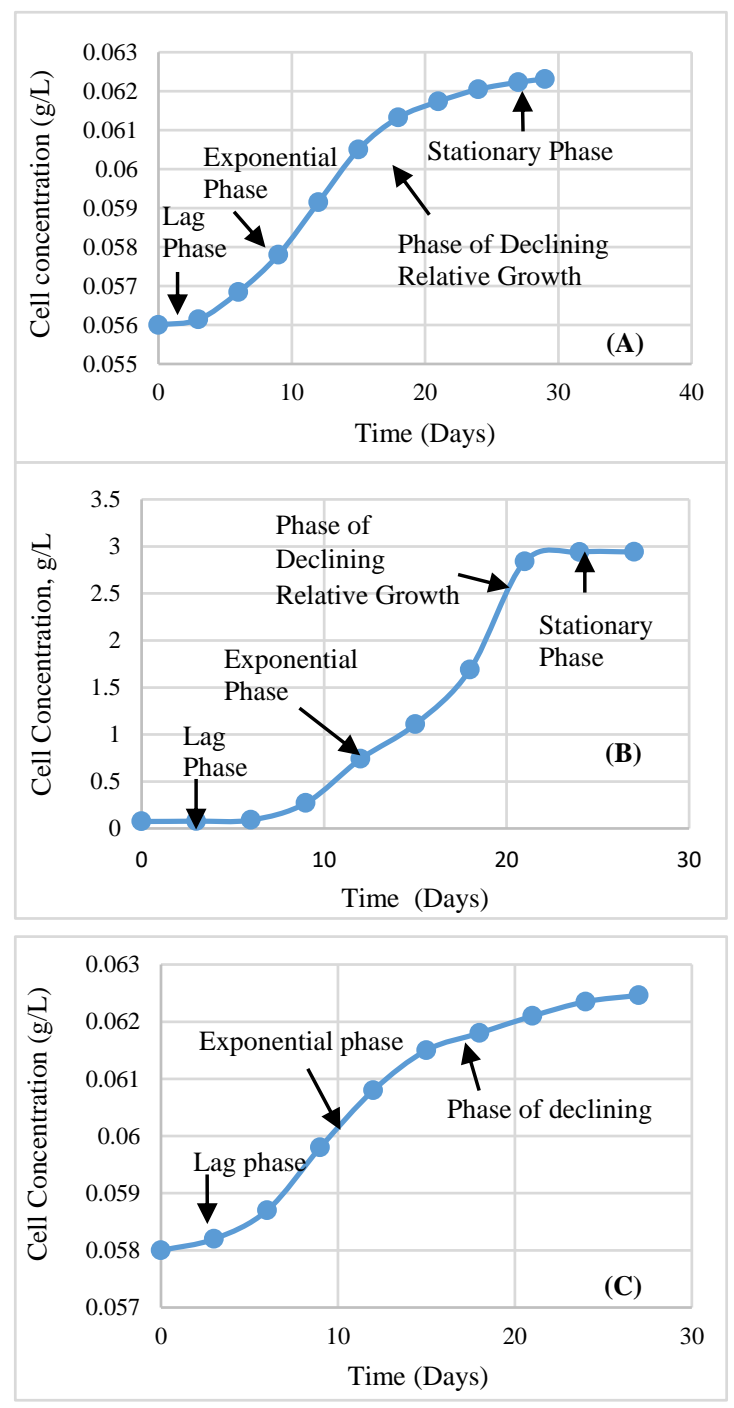

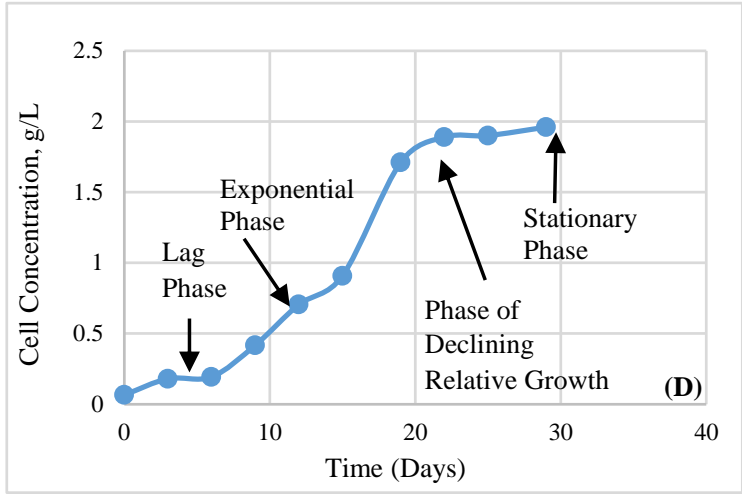

Fig. 4 Growth curves for (A) $\mathrm{CH}$ medium (B) Modified $\mathrm{CH}$ medium (C) BB medium (D) Modified BB medium

\subsection{Duration of exponential phase}

Using the growth curves, duration of exponential phase for each medium can be determined. Figure 5 represents the duration of exponential phase for each medium. It also shows the concentration of Chlorella vulgaris at the end of each exponential phase. It can be seen that modified $\mathrm{CH}$ medium has comparatively small exponential phase with highest cell concentration.

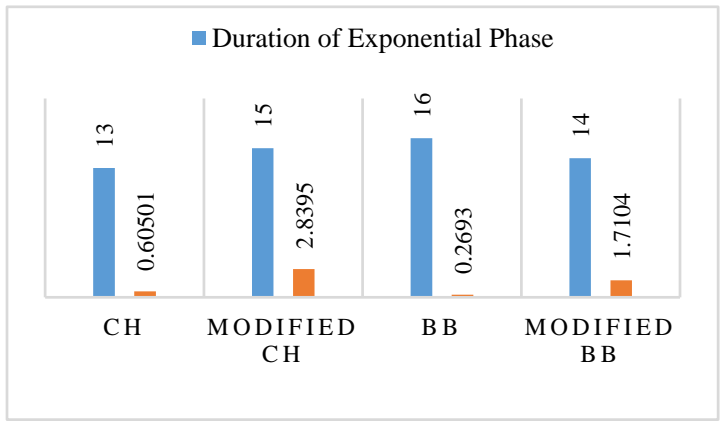

Fig.5 Properties of exponential phase

\subsection{Determination of overall specific growth rate}

Using the cell concentrations, overall specific growth rate for each medium was calculated. The obtained values were $0.0039 \mathrm{~d}^{-1}, 0.136 \mathrm{~d}^{-1}, 0.0028 \mathrm{~d}^{-1}$ and $0.041 \mathrm{~d}^{-1}$ respectively for $\mathrm{CH}$, modified $\mathrm{CH}, \mathrm{BB}$ and modified BB medium.

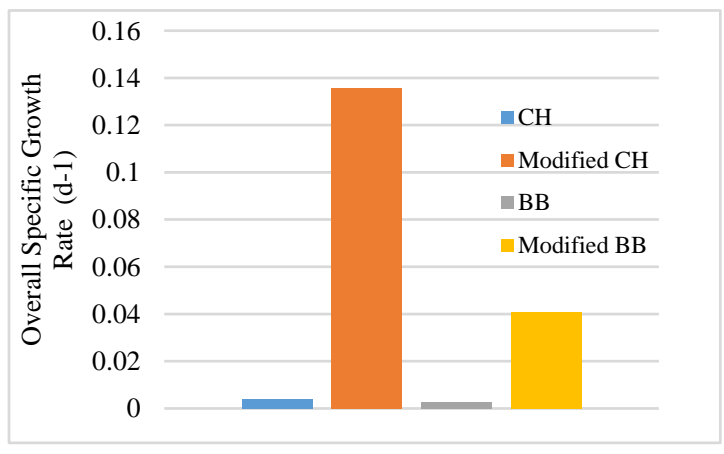

Fig.6 Overall specific growth rates in $\mathrm{d}^{-1}$ for four media 
Figure 6 shows a comparative representation of the values of overall specific growths for all four media. It can also be seen that the value is the highest for modified $\mathrm{CH}$ medium.

\subsection{Determination of doubling time}

Doubling time or the time required for the cells to divide for $\mathrm{CH}$, modified $\mathrm{CH}, \mathrm{BB}$ and modified $\mathrm{BB}$ are 1847.57 hours, 55.27 hours, 2636.49 hours and 177.26 hours respectively. Figure 7 is a comparative representation of the values of doubling time for all four media.

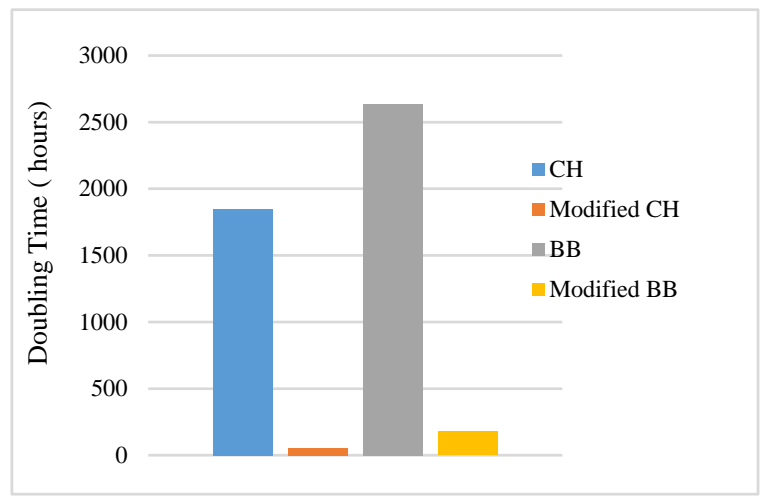

Fig.7 Doubling time in hours for four media

\subsection{Biomass productivity}

Biomass productivity represents which culture media yields the maximum biomass from $1 \mathrm{~L}$ of culture per day. The respective values for algal biomass productivity for $\mathrm{CH}$, modified $\mathrm{CH}, \mathrm{BB}$ and modified $\mathrm{BB}$ are $0.0002 \mathrm{gL}^{-1} \mathrm{~d}^{-1}, 0.106 \mathrm{gL}^{-1} \mathrm{~d}^{-1}$, $0.00017 \mathrm{gL}^{-1} \mathrm{~d}^{-1}$ and $0.005 \mathrm{gL}^{-1} \mathrm{~d}^{-1}$. Figure 8 shows the comparison among the values.

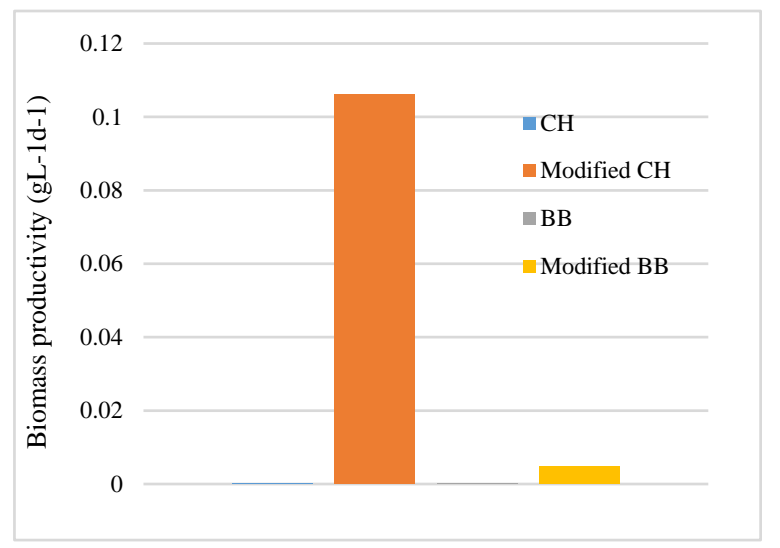

Fig.8 Biomass productivity in gL-1d-1 for four media

\subsection{Selection of suitable media}

After comparing the values of all four parameters, it can be seen that the microalgae Chlorella vulgaris responded the best in modified $\mathrm{CH}$ medium.

\section{Conclusion}

As an initiative to introduce a new strain of microalgae with high lipid content in the local environment and grow it in mass scale, it is significant to find a suitable medium at first. This study has led to a better understanding of the behaviors of the microalgae Chlorella vulgaris in different media. The study also showed that Bold's Basal medium which is the most frequently used medium for culturing Chlorella vulgaris did not respond satisfactorily in our local environment. However, the locally developed medium $\mathrm{CH}$ along with vitamin mixture has shown good response. This study was conducted as foundation to produce algal biodiesel in local environment. Understanding the growth kinetics of the microalgae is a crucial part of the algal biodiesel production process. Optimization of growth kinetics will help to develop realistic technical and commercial model for biodiesel production from microalgae.

\section{Acknowledgment}

This research was supported by BCEF Academic Research Fund and CASR Research Fund.

\section{References}

[1] Sacasa Castellanos, C., Batch and Continuous Studies of Chlorella vulgaris in Photo- Bioreactors, in Chemical and Biochemical Engineering. 2013, University of Ottowa: Canada.

[2] Bark, M., Cultivation of Eleven Different Species of Freshwater Microalgae Using Simulated Flue Gas Mimicking Effluents from Paper Mills as Carbon Source, in Department of Chemical and Biological Engineering. 2012, Chalmers University of Technology

[3] Schenk, P.M., Thomas-Hall, S.R., Stephens, E. et al., Second Generation Biofuels: High-Efficiency Microalgae for Biodiesel Production. BioEnergy Research, 2008. 1: p. 20-43.

[4] Srivastava, A., and Prasad, R., Triglycerides-based diesel fuels. Renewable and Sustainable Energy Reveiws, 2000. 4(2): p. 111-133.

[5] Feng, P., Z., Deng, L. Fan and Z. Hu, Lipid accumulation and growth characteristics of Chlorella zofingiensis under different nitrate and phosphate concentrations. Journal of Bioscience and Bioengineering, 2012. 114: p. 405-410.

[6] Subramanium, R., Dufreche S., Zappi, M., and Bajpai, R. , Microbial lipids from renewable resources: prodction and characterization. Journal of Industrial Microbiology and Biotechnology, 2010. 37: p. 12711287.

[7] Chisti, Y., Biodiesel from microalgae Biotechnology Advance, 2007. 25(3): p. 294-306. 
[8] Demirbas, A., Progress and recent trends in biodiesel fuels. Energy Conversion Management, 2009. 50: p. 14-34.

[9] Viswanath, B., Mutanda, T., White, S., and Bux F., The microalgae- a future source of biodiesel. Dynamic Biochemistry, Process Biotechnology and Molecular Biology, 2010. 4(1): p. 37-47.

[10] Anderson, R.A., Measuring Growth Rates in Microalgal Cultures, in Algal Culturing Techniques. 2005. p. 269-276.

[11] Bartosh, Y., Banks, C.J., Algal growth response and survival in a range of light and temperature conditions: implications for non-steady-state conditions in waste stabilisation ponds. Water Sci Technol. 2007;55(11):211-8. doi: 10.2166/wst.2007.365. PMID: 17591214.
[12] Janta, K., Pekkoh, J., Tongsiri, S., Pumas, C., and Peerapornpisai, Y., Selection of Some Native Microalgal Strains for Possibility of Bio-oil Production in Thailand. Chiang Mai Journal of Science, 2013. 40(4): p. 593-602.

[13] Hempel, N., Petrick, I., and Behrendt, F., Biomass productivity and productivity of fatty acids and amino acids of microalgae strains as key characteristics of suitability for biodiesel production. Journal of Applied Phycology, 2012. 24: p. 1407-1418.

(C)Bangladesh Uni. of Engg. \& Tech. 